\title{
ON THE $q$-ANALOGUE OF LAPLACE TRANSFORM
}

\author{
WON SANG CHUNG ${ }^{1}$ AND TAEKYUN KIM ${ }^{2}$
}

\begin{abstract}
In this paper, we consider a $q$-analogue of Laplace transform and we investigate some properties of $q$-Laplace transform. From our investigation, we derive some interesting formulae related to $q$-Laplace transform.
\end{abstract}

\section{INTRODUCTION}

For $q \in[0,1]$, we define the $q$-Shifted factorials by $(a: q)_{0}=1,(a: q)_{n}=$ $\prod_{i=0}^{n-1}\left(1-a q^{i}\right)$, and $(a: q)_{\infty}=\lim _{n \rightarrow \infty}(a: q)_{n}=\prod_{i=0}^{\infty}\left(1-a q^{i}\right)$. If $x$ is classical object, such as a complex number, its $q$-version is defined by $[x]_{q}=\frac{1-q^{x}}{1-q}$. As is well-known, the $q$-exponential functions are given by

$$
\left.E_{q}(-z)=((1-q) z: q)\right)_{\infty}=\sum_{n=0}^{\infty} \frac{(-1)^{n} q^{\left(\begin{array}{c}
n \\
2
\end{array}\right)}}{[n]_{q} !} z^{n}
$$

and

$$
e_{q}(z)=\frac{1}{((1-q) z: q)_{\infty}}=\sum_{n=0}^{\infty} \frac{1}{[n]_{q} !} z^{n}, \quad(\text { see }[1-8]),
$$

where $[n]_{q} !=[n]_{q}[n-1]_{q} \cdots[2]_{q}[1]_{q}$.

For $t, x, y \in \mathbb{R}$ and $n \in \mathbb{Z} \geq 0$, the $q$-binomial theorem is also given by

$$
\begin{gathered}
(x+y)_{q}^{n}=\prod_{j=0}^{n-1}\left(x+q^{j} y\right)=\sum_{k=0}^{n}\left(\begin{array}{l}
n \\
k
\end{array}\right)_{q} x^{n-k} q^{\left(\begin{array}{l}
2 \\
k
\end{array}\right)} y^{k}, \\
(1+x)_{q}^{t}=\frac{(x: q)_{\infty}}{\left(q^{t} x: q\right)_{\infty}}, \text { and } \frac{1}{(x-y)_{q}^{n}}=\sum_{k=0}^{\infty}\left(\begin{array}{c}
n+k-1 \\
k
\end{array}\right)_{q} x^{n-k} y^{k}
\end{gathered}
$$

where

$$
\left(\begin{array}{l}
n \\
k
\end{array}\right)_{q}=\frac{[n]_{q} !}{[n-k]_{q} ![k]_{q} !}=\frac{[n]_{q}[n-1]_{q} \cdots[n-k+1]_{q}}{[k]_{q} !}, \quad(\text { see }[2,6]) .
$$

From (1.4), we note that

$$
(1+x)_{q}^{s+t}=(1+x)_{q}^{s}\left(1+q^{s} x\right)_{q}^{t} .
$$

For $s \in \mathbb{C}$ with $s>0$, the gamma function is defined by

$$
\Gamma(s)=\int_{0}^{\infty} e^{-t} t^{s-1} d t .
$$

1991 Mathematics Subject Classification. 05A40, 05A19.

Key words and phrases. Frobenius-type Eulerian polynomials, Frobenius-type Eulerian numbers, umbral calculus. 
By (1.6), we get

$$
\Gamma(s+1)=s \Gamma(s), \Gamma(n+1)=n !,(n \in \mathbb{N}) .
$$

The Jackson q-derivative is defined by

$$
D_{q} f(x)=\frac{\partial_{q} f(x)}{\partial_{q} x}=\frac{f(x)-f(q x)}{(1-q) x},(\text { see }[1-8]) .
$$

Note that $\lim _{q \rightarrow 1} D_{q} f(x)=f^{\prime}(x)$. The definite Jackson q-integral is given by

$$
\int_{0}^{x} f(t) d_{q} t=(1-q) \sum_{a=0}^{\infty} f\left(q^{a} x\right) x q^{a}, \quad \text { (see [3-4]). }
$$

Thus, by (1.7) and (1.8), we get

$$
\int_{0}^{x} \frac{\partial_{q}}{\partial_{q} t} f(t) d_{q} t=f(x)-f(0) .
$$

The improper $q$-integral of $f$ is given by

$$
\int_{0}^{\frac{\infty}{a}} f(x) d_{q} x=(1-q) \sum_{n \in \mathbb{Z}} \frac{q^{n}}{a} f\left(\frac{q^{n}}{a}\right) .
$$

Thus, by (1.10), we get

$$
\begin{aligned}
\int_{x}^{\infty} f(y) d_{q} y & =\int_{0}^{x \cdot \infty} f(y) d_{1} y-\int_{0}^{x} f(y) d_{q} y \\
& =x(1-q) \sum_{n=0}^{\infty} q^{-n} f\left(q^{-n} x\right) .
\end{aligned}
$$

Let $f$ be a function defined for $t \geq 0$. Then the integral

$$
\mathcal{L}(f(t))=\int_{0}^{\infty} e^{-s t} f(t) d t, \quad \text { see [9]) }
$$

is said to be the Laplace transform of $f$, provided the integral converges.

In this paper, we consider a $q$-analogue of Laplace transform, which is called by $q$-Laplace transform, and we investigate some properties of $q$-Laplace transform. From our investigation, we derive some interesting formulae related to $q$-Laplace transform.

\section{2. $q$-LAPLACE TRANSFORMS}

Now we consider two types of the $q$-Laplace transform. The $q$-Laplace transform of the first kind is defined by

$$
F(s)=L_{q}(f(t))=\int_{0}^{\infty} E_{q}(-q s t) f(t) d_{q}(t),(s>0) .
$$

Then, by (2.1), we get

$$
\left.L_{q}(\alpha f(t)+\beta g(t))=\alpha L_{q}(f(t))+\beta_{q} L g(t)\right),
$$

where $\alpha, \beta$ are constants.

The q-extension of gamma function is defined by

$$
\Gamma_{q}(t)=\int_{0}^{\infty} x^{t-1} E_{q}(-q x) d_{q} x,(t>0), \quad(\text { see }[2,6]) .
$$


Thus, by (2.3), we get

$$
\Gamma_{q}(t+1)=[t]_{q} \Gamma_{q}(t), \Gamma_{q}(n+1)=[n]_{q} !, \quad(\text { see }[2,6]) .
$$

From (1.2), we can derive

$$
e_{q}(i z)=\sum_{n=0}^{\infty} \frac{i^{n} z^{n}}{[n]_{q} !}=\sum_{n=0}^{\infty} \frac{(-1)^{n}}{[2 n]_{q} !} z^{2 n}+i \sum_{n=0}^{\infty} \frac{(-1)^{n} z^{2 n+1}}{[2 n+1]_{q} !} .
$$

By (2.5), we define $q$-cosine and $q$-sine function as follows:

$$
\cos _{q}(z)=\sum_{n=0}^{\infty} \frac{(-1)^{n} z^{2 n}}{[2 n]_{q} !}=\frac{1}{2}\left(e_{q}(i z)+e_{q}(-i z)\right),
$$

and

$$
\sin _{q}(z)=\sum_{n=0}^{\infty} \frac{(-1)^{n}}{[2 n+1]_{q} !}=\frac{1}{2 i}\left(e_{q}(i z)-e_{q}(-i z)\right),
$$

where $i=\sqrt{-1}$. From (2.1) and (2.4), we note that

$$
\begin{gathered}
L_{q}(1)=\frac{1}{s}(s>0), L_{q}(t)=\frac{1}{s^{2}}(s>0), L_{q}\left(e_{q}(-3 t)\right)=\frac{1}{s+3}(s>-3), \\
L_{q}\left(\sin _{q} 2 t\right)=\frac{2}{s^{2}+4}, L_{q}(1+5 t)=\frac{1}{s}+\frac{5}{s^{2}}(s>0), \cdots .
\end{gathered}
$$

We state the generalization of some of the preceding examples by mean of the next theorem. From this point on we shall also refrain from stating any restrictions on $s$; it is understood that $s$ is sufficiently restricted to guarantee the convergence of the appropriate $q$-Laplace transform.

For $\alpha \in \mathbb{R}$ with $\alpha>-1$, we have

$$
\begin{aligned}
L_{q}\left(t^{\alpha}\right) & =\int_{0}^{\infty} E_{q}(-q s t) t^{\alpha} d_{q} t=\frac{1}{s^{\alpha+1}} \int_{0}^{\infty} E_{q}(-q t) t^{\alpha} d_{q} t \\
& =\frac{1}{s^{\alpha+1}} \Gamma_{q}(\alpha+1) .
\end{aligned}
$$

In particular, $\alpha=n \in \mathbb{N}$, by (2.8), we get

$$
L_{q}\left(t^{n}\right)=\frac{1}{s^{n+1}} \Gamma_{q}(n+1)=\frac{[n]_{q} !}{s^{n+1}} .
$$

Let us take $\alpha=-\frac{1}{2}$ and $\alpha=\frac{1}{2}$. Then we see that

$$
L_{q}\left(t^{-\frac{1}{2}}\right)=\frac{1}{s^{\frac{1}{2}}} \Gamma_{q}\left(\frac{1}{2}\right)=\frac{1}{\sqrt{s}} \Gamma_{q}\left(\frac{1}{2}\right),
$$

and

$$
L_{q}\left(t^{\frac{1}{2}}\right)=\frac{1}{s^{\frac{3}{2}}} \Gamma_{q}\left(\frac{3}{2}\right)=\frac{\Gamma_{q}\left(\frac{1}{2}\right)}{[2]_{q^{\frac{1}{2}}} \sqrt{s^{3}}} .
$$


By (1.1) and (1.2), we get

$$
\begin{aligned}
L_{s}\left(e_{q}(a t)\right) & =\int_{0}^{\infty} E_{q}(-q s t) e_{q}(a t) d_{q} t \\
& =\sum_{n=0}^{\infty} \frac{a^{n}}{[n]_{q} !} \int_{0}^{\infty} E_{q}(-q s t) t^{n} d_{q} t \\
& =\sum_{n=0}^{\infty} \frac{a^{n}}{[n]_{q} !} \frac{\Gamma_{q}(n+1)}{s^{n+1}}=\frac{1}{s} \sum_{n=0}^{\infty}\left(\frac{a}{s}\right)^{n} \\
& =\frac{1}{s}\left(\frac{1}{1-\frac{a}{s}}\right)=\frac{1}{s-a},
\end{aligned}
$$

and

$$
\begin{aligned}
L_{q}\left(E_{q}(a t)\right) & =\int_{0}^{\infty} E_{q}(-q s t) E_{q}(a t) d_{q} t \\
& =\sum_{n=0}^{\infty} \frac{(-1)^{n} q^{\left(\begin{array}{c}
n \\
2
\end{array}\right)} a^{n}}{[n]_{q} !} \int_{0}^{\infty} E_{q}(-q s t) t^{n} d_{q} t \\
& =\sum_{n=0}^{\infty} \frac{(-1)^{n}}{[n]_{q} !} q^{\left(\begin{array}{c}
n \\
2
\end{array}\right)} a^{n} \frac{\Gamma_{q}(n+1)}{s^{n+1}}=\sum_{n=0}^{\infty}(-1)^{n} q^{\left(\begin{array}{c}
n \\
2
\end{array}\right)} \frac{a^{n}}{s^{n+1}} .
\end{aligned}
$$

Therefore, by (2.8)-(2.13), we obtain the following theorem.

Theorem 2.1. For $\alpha \in \mathbb{R}$ with $\alpha>-1$, we have

$$
L_{q}\left(t^{\alpha}\right)=\frac{1}{s^{n+1}} \Gamma_{q}(\alpha+1) .
$$

In particular, $\alpha=n \in \mathbb{N}$, we get

$$
L_{q}\left(t^{n}\right)=\frac{[n]_{q} !}{s^{n+1}} .
$$

Moreover,

$$
L_{q}\left(t^{-\frac{1}{2}}\right)=\frac{1}{\sqrt{s}} \Gamma_{q}\left(\frac{1}{2}\right), L_{q}\left(t^{\frac{1}{2}}\right)=\frac{\Gamma_{q}\left(\frac{1}{2}\right)}{[2]_{q^{\frac{1}{2}}} \sqrt{s^{3}}}
$$

and

$$
L_{s}\left(e_{q}(a t)\right)=\frac{1}{s-a}, L_{q}\left(E_{q}(a t)\right)=\sum_{n=0}^{\infty}(-1)^{n} q^{\left(\begin{array}{c}
n \\
2
\end{array}\right)} \frac{a^{n}}{s^{n+1}} .
$$

From (2.6) and (2.7), we have

$$
\begin{aligned}
L_{q}\left(\cos _{q} a t\right) & =\sum_{n=0}^{\infty} \frac{(-1)^{n} a^{2 n}}{[2]_{q} !} \int_{0}^{\infty} E_{q}(-q s t) t^{2 n} d_{q} t \\
& =\sum_{n=0}^{\infty} \frac{(-1)^{n} a^{2 n}}{[2 n]_{q} !} \frac{1}{s^{2 n+1}} \Gamma_{q}(2 n+1) \\
& =\frac{1}{s} \sum_{n=0}^{\infty}(-1)^{n}\left(\frac{a}{s}\right)^{2 n}=\frac{1}{s} \frac{1}{1+\left(\frac{a}{s}\right)^{2}}=\frac{s}{s^{2}+a^{2}},
\end{aligned}
$$


and

$$
\begin{aligned}
L_{q}\left(\sin _{q} a t\right) & =\sum_{n=0}^{\infty} \frac{(-1)^{n} a^{2 n+1}}{[2 n+1]_{q} !} \int_{0}^{\infty} E_{q}(-q s t) t^{2 n+1} d_{q} t \\
& =\sum_{n=0}^{\infty} \frac{(-1)^{n} a^{2 n+1}}{[2 n+1]_{q} !} \frac{\Gamma_{q}(2 n+2)}{s^{2 n+2}} \\
& =\frac{1}{s} \sum_{n=0}^{\infty}(-1)^{n}\left(\frac{a}{s}\right)^{2 n+1}=\frac{1}{s} \frac{\frac{a}{s}}{1+\left(\frac{a}{s}\right)^{2}}=\frac{a}{s^{2}+a^{2}} .
\end{aligned}
$$

Let us define hyperbolic q-cosine and hyperbolic q-sine functions as follows:

$$
\cosh _{q} t=\frac{e_{q}(t)+e_{q}(-t)}{e}, \sinh _{q} t=\frac{e_{q}(t)-e_{q}(-t)}{2} .
$$

From (2.1), (2.12) and (2.16), we note that

$$
\begin{aligned}
L_{q}\left(\cosh _{q} a t\right) & =\frac{1}{2}\left\{L_{q}\left(e_{q}(a t)\right)+L_{q}\left(e_{q}(-a t)\right)\right\} \\
& =\frac{1}{2}\left\{\frac{1}{s-a}+\frac{1}{s+a}\right\}=\frac{s}{s^{2}-a^{2}},
\end{aligned}
$$

and

$$
\begin{aligned}
L_{q}\left(\sinh _{q} a t\right) & =\frac{1}{2}\left\{L_{q}\left(e_{q}(a t)\right)-L_{q}\left(e_{q}(-a t)\right)\right\} \\
& =\frac{1}{2}\left\{\frac{1}{s-a}-\frac{1}{s+a}\right\}=\frac{a}{s^{2}-a^{2}} .
\end{aligned}
$$

Therefore, by (2.14)-(2.18), we obtain the following theorem.

Theorem 2.2. (Transforms of q-trigonometric function)

$$
\begin{aligned}
L_{q}\left(\cos _{q} a t\right) & =\frac{s}{s^{2}+a^{2}}, L_{q}\left(\sin _{q} a t\right)=\frac{a}{s^{2}+a^{2}} \\
L_{q}\left(\cosh _{q} a t\right) & =\frac{s}{s^{2}-a^{2}}, L_{q}\left(\sinh _{q} a t\right)=\frac{a}{s^{2}-a^{2}} .
\end{aligned}
$$

Now, we observe that

$$
\begin{aligned}
E_{q}(-q s t) & =\sum_{n=0}^{\infty} \frac{(-1)^{n} q^{\left(\begin{array}{c}
n \\
2
\end{array}\right)}}{[n]_{q} !} q^{n} s^{n} t^{n}=\sum_{n=0}^{\infty} \frac{(-1)^{n} q^{\left(\begin{array}{c}
n \\
2
\end{array}\right)}}{[n]_{q} !}\left([n]_{q}(q-1)+1\right) s^{n} t^{n} \\
& =(q-1) \sum_{n=1}^{\infty} \frac{(-1)^{n} q^{\left(\begin{array}{c}
n \\
2
\end{array}\right)}}{[n-1]_{q} !} s^{n} t^{n}+\sum_{n=0}^{\infty} \frac{(-1)^{n} q^{\left(\begin{array}{c}
n \\
2
\end{array}\right)}}{[n]_{q} !} s^{n} t^{n} \\
& =-(q-1) s t \sum_{n=0}^{\infty} \frac{(-1)^{n} q^{\left(\begin{array}{c}
n \\
2
\end{array}\right)}}{[n]_{q} !} q^{n} s^{n} t^{n}+\sum_{n=0}^{\infty} \frac{(-1)^{n} q^{\left(\begin{array}{c}
n \\
2
\end{array}\right)}}{[n]_{q} !} s^{n} t^{n} \\
& =-(q-1) s t E_{q}(-q s t)+E_{q}(-s t) .
\end{aligned}
$$

Thus, by (2.19), we get

$$
\begin{aligned}
E_{q}(-q s t) & =\frac{1}{1+(q-1) s t} E_{q}(-s t)=\frac{1}{(1+(q-1) s t)\left(1+(q-1) q^{-1} s t\right)} E_{q}\left(-q^{-1} s t\right) \\
& =\cdots=\frac{1}{(1+(q-1) s t)\left(1+(q-1) q^{-1} s t\right) \cdots\left(1+(q-1) q^{-k} s t\right)} E_{q}\left(-q^{-k} s t\right) .
\end{aligned}
$$


Now, we dicuss the $q$-differential equation. The main purpose of $q$-Laplace transform is in converting $q$-differential equation into simpler form which may be solved more easily. Like the ordinary Laplace transform, we can compute the $q$-Laplace transform of derivative by using the definition of $q$-Laplace transform.

Now, we oberve that

$$
\begin{aligned}
D_{q}(f(t) g(t)) & =\frac{\partial}{\partial_{q} t}(f(t) g(t))=\frac{\partial f(t)}{\partial_{q} t} g(t)+f(q t) \frac{\partial g(t)}{\partial_{q} t} \\
& =\left(D_{q} f(t)\right) g(t)+f(q t)\left(D_{q} g(t)\right) .
\end{aligned}
$$

Thus, by (2.21), we get

$$
\int_{0}^{\infty}\left(D_{q} f(t)\right) g(t) d_{q} t=\int_{0}^{\infty} D_{q}(f(t) g(t)) d_{q} t-\int_{0}^{\infty} f(q t)\left(D_{q} g(t)\right) d_{q} t .
$$

It is easy to show that

$$
\begin{aligned}
D_{q} E_{q}(-q s t) & =\frac{\partial}{\partial_{q} t} \sum_{n=1}^{\infty} \frac{q^{\left(\begin{array}{c}
n \\
2
\end{array}\right)}(-1)^{n}}{[n]_{q} !} q^{n} s^{n} t^{n}=\sum_{n=1}^{\infty} \frac{q^{\left(\begin{array}{c}
n \\
2
\end{array}\right)(-1)^{n}}}{[n-1]_{q} !} q^{n} s^{n} t^{n-1} \\
& =-s \sum_{n=0}^{\infty} \frac{q^{\left(\begin{array}{c}
n \\
2
\end{array}\right)}(-1)^{n}}{[n]_{q} !} q^{n+1} s^{n} t^{n} .
\end{aligned}
$$

From (2.20), (2.22) and (2.23), we have

$$
\begin{aligned}
L_{q}\left(D_{q} f(t)\right) & =\int_{0}^{\infty}\left(D_{q} f(t)\right) E_{q}(-q s t) d_{q} t=-f(0)-\int_{0}^{\infty} f(q t) \frac{\partial}{\partial_{q} t} E_{q}(-q s t) d_{q} t \\
& =-f(0)+s \sum_{n=0}^{\infty} \frac{q^{\left(\begin{array}{c}
n+1 \\
2
\end{array}\right)}}{[n]_{q} !}(-1)^{n} \int_{0}^{\infty} f(q t) q^{n+1} s^{n} t^{n} d_{q} t \\
& =-f(0)+s \int_{0}^{\infty} f(t) \sum_{n=0}^{\infty} \frac{(-1)^{n} q^{\left(\begin{array}{c}
n \\
2
\end{array}\right)}}{[n]_{q} !}(q s t)^{n} d_{q} t \\
& =-f(0)+s \int_{0}^{\infty} f(t) E_{q}(-q s t) d_{q} t=-f(0)+s L_{q}(f(t)) .
\end{aligned}
$$

If we replace $f(t)$ by $D_{q} f(t)$, we see that

$$
\begin{aligned}
L_{q}\left(D_{q}^{2} f(t)\right) & =-\left(D_{q} f\right)(0)+s \int_{0}^{\infty}\left(D_{q} f(t)\right) E_{q}(-q(t)) d_{q} t \\
& =-\left(D_{q} f\right)(0)+s L_{q}\left(D_{q} f(t)\right) \\
& =-\left(D_{q} f\right)(0)+s(-f(0))+s L_{q}(f(t)) \\
& =-\left(D_{q} f\right)(0)-s f(0)+s^{2} L_{q}(f(t)) .
\end{aligned}
$$

Continuing this process, we get

$$
L_{q}\left(f^{(n)}(t)\right)=s^{n} L_{q}(f(t))-\sum_{i=0}^{n-1} s^{n-1-i} f^{(i)}(0),
$$

where $f^{(n)}(t)=\left(\frac{\partial_{q}}{\partial_{q} t}\right)^{n} f(t)=D_{q}^{n} f(t), f^{(n)}(0)=\left.f^{(n)}(t)\right|_{t=0}$.

A function $f$ is said to be of exponential order $c$ if there exists $c, M>0$ and $T>0$ such that

$$
|f(t)| \leq M e^{c t} \text { for all } t>T \text {. }
$$


If $f(t)$ is piecewise continuous on the interval $(0, \infty)$ and of exponential order $c$, then $L_{q}(f(t))$ exists for $s>c$. Therefore, by (2.26), we obtain the following theorem.

Theorem 2.3. If $f, f^{\prime}, \cdots, f^{(n-1)}$ are continuous on $(0, \infty)$ and are of exponential order and if $f^{(n)}(t)$ is piecewise continunus on $(0, \infty)$, then we have

$$
L_{q}\left(f^{(n)}(t)\right)=s^{n} L_{q}(f(t))-\sum_{i=0}^{n-1} s^{n-1-i} f^{(i)}(0),
$$

where $f^{(n)}(t)=\left(\frac{\partial_{q}}{\partial_{q} t}\right)^{n} f(t)$.

Let us consider the following $q$-derivative in $s$ :

$$
\begin{aligned}
\frac{\partial F\left(q^{-1} s\right)}{\partial_{q} s} & =\int_{0}^{\infty}\left(\frac{\partial_{q}}{\partial_{q} s} E_{q}(-s t)\right) f(t) d_{q} t \\
& =-\int_{0}^{\infty} t E_{q}(-q s t) f(t) d_{q} t \\
& =-L_{q}(t f(t)),
\end{aligned}
$$

and

$$
\begin{aligned}
q\left(\frac{\partial_{q}}{\partial_{q} s}\right)^{2} F\left(q^{-2} s\right) & =q\left(\frac{\partial_{q}}{\partial_{q} s}\right)^{2} \int_{0}^{\infty} E_{q}\left(-q^{-1} s t\right) f(t) d_{q} t \\
& =q \int_{0}^{\infty}\left(\left(\frac{\partial_{q}}{\partial_{q} s}\right)^{2} E_{q}\left(-q^{-1} s t\right)\right) f(t) d_{q} t \\
& =q \sum_{n=0}^{\infty} \frac{(-1)^{n+2}}{[n]_{q} !} q^{\left(\begin{array}{c}
n \\
2
\end{array}\right)} q^{n-1} s^{n} \int_{0}^{\infty} t^{n+2} f(t) d_{q} t \\
& =\int_{0}^{\infty}(-1)^{2}\left(\sum_{n=0}^{\infty} \frac{(-1)^{n} q^{\left(\begin{array}{c}
n \\
2
\end{array}\right)}}{[n]_{q} !} q^{n} s^{n} t^{n}\right) t^{2} f(t) d_{q} t \\
& =(-1)^{2} \int_{0}^{\infty} E_{q}(-q s t) t^{2} f(t) d_{q} t=(-1)^{2} L_{q}\left(t^{2} f(t)\right) .
\end{aligned}
$$

Continuing this process, we get

$$
L_{q}\left(t^{n} f(t)\right)=(-1)^{n} q^{\left(\begin{array}{c}
n \\
2
\end{array}\right)}\left(\frac{\partial_{q}}{\partial_{q} s}\right)^{n} F\left(q^{-n s}\right) .
$$

Therefore, by (2.29), we obtain the following theorem.

Theorem 2.4 ( $q$-Derivative of Transforms). For $n \in \mathbb{N}$, we have

$$
L_{q}\left(t^{n} f(t)\right)=(-1)^{n} q^{\left(\begin{array}{c}
n \\
2
\end{array}\right)}\left(\frac{\partial_{q}}{\partial_{q} s}\right)^{n} F\left(q^{-n} s\right) .
$$


From Theorem 2.4 we note that

$$
\begin{aligned}
L_{q}\left(t^{n} e_{q}(a t)\right) & =(-1)^{n} q^{\left(\begin{array}{c}
n \\
2
\end{array}\right)}\left(\frac{\partial_{q}}{\partial_{q} s}\right)^{n} \int_{0}^{\infty} E_{q}\left(-q^{-n+1} s t\right) e_{q}(a t) d_{q} t \\
& =(-1)^{n} q^{\left(\begin{array}{c}
n \\
2
\end{array}\right)}\left(\frac{\partial_{q}}{\partial_{q} s}\right)^{n}\left(\frac{1}{q^{-n} s-a}\right) \\
& =\frac{(-1)^{n} q^{\left(\begin{array}{c}
n \\
2
\end{array}\right)}[n]_{q} !(-1)^{n} q^{-n^{2}}}{(s-a)\left(q^{-1} s-a\right) \cdots\left(q^{-n} s-a\right)} \\
& =\frac{q^{-\left(\begin{array}{c}
n+1 \\
2
\end{array}\right)[n]_{q} !}}{(s-a)\left(q^{-1} s-a\right) \cdots\left(q^{-n} s-a\right)},
\end{aligned}
$$

and

$$
\begin{aligned}
L_{q}\left(e_{q}(a t) f(t)\right) & =\sum_{n=0}^{\infty} \frac{a^{n}}{[n]_{q} !} L_{q}\left(t^{n} f(t)\right) \\
& =\sum_{n=0}^{\infty} \frac{a^{n}}{[n]_{q} !}(-1)^{n} q^{\left(\begin{array}{c}
n \\
2
\end{array}\right)}\left(\frac{\partial_{q}}{\partial_{q} s}\right)^{n} F\left(q^{-n} s\right) \\
& =\sum_{n=0}^{\infty} \frac{(-a)^{n}}{[n]_{q} !} q^{\left(\begin{array}{c}
n \\
2
\end{array}\right)}\left(\frac{\partial_{q}}{\partial_{q} s}\right)^{n} F\left(q^{-n} s\right) .
\end{aligned}
$$

Therefore, by (2.30) and (2.31), we obtain the following corollary.

Corollary 2.5. For $n \in \mathbb{N}$, we have

$$
L_{q}\left(e_{q}(a t) f(t)\right)=\sum_{n=0}^{\infty} \frac{(-a)^{n}}{[n]_{q} !} q^{\left(\begin{array}{c}
n \\
2
\end{array}\right)}\left(\frac{\partial_{q}}{\partial_{q} s}\right)^{n} F\left(q^{-n} s\right) .
$$

In particular, for $f(t)=t^{n}(n \in \mathbb{N})$, we have

$$
L_{q}\left(t^{n} e_{q}(a t)\right)=\frac{q^{-\left(\begin{array}{c}
n+1 \\
2
\end{array}\right)}[n]_{q} !}{(s-a)\left(q^{-1} s-a\right) \cdots\left(q^{-n} s-a\right)} .
$$

For $t, s>0$, the $q$-beta function is defined by

$$
B_{q}(t, s)=\int_{0}^{1} x^{t-1}(1-q x)_{q}^{s-1} d_{q} x, \quad(\text { see }[2,6]) .
$$

From (2.32), we note that

$$
B_{q}(t, s)=\frac{\Gamma_{q}(t) \Gamma_{q}(s)}{\Gamma_{q}(t+s)},(\text { see }[2,6]) .
$$

It is easy to show that

$$
T_{q}^{\alpha}(f)=\int_{0}^{t}(t-q s)_{q}^{\alpha-1} f(s) d_{q} s=t \int_{0}^{1}(t-q r t)_{q}^{\alpha-1} f(r t) d_{q} r, \quad(\alpha>0) .
$$

In particular, if we take $f(t)=t^{\beta}(\beta>0)$, then

$$
\begin{aligned}
\int_{0}^{t}(t-q s)_{q}^{\alpha-1} t^{\beta} d_{q} s & =t^{\alpha+\beta} \int_{0}^{1}(1-q r)_{q}^{\alpha-1} r^{\beta} d_{q} r \\
& =B_{q}(\alpha, \beta+1) t^{\alpha+\beta}
\end{aligned}
$$


If functions $f$ and $g$ are piecewise continuous on $(0, \infty)$, then a special product, denote by $f * g$ is defined by the integral

$$
(f * g)(t)=\int_{0}^{t} f(\tau) g(t-\tau) d \tau
$$

and is called convolution of $f$ and $g$.

Now, we consider the $q$-analogue of convolution of $f$ and $g$.

Let $f_{1}(t)=t^{\alpha}, g(t)=t^{\beta-1}(\alpha, \beta>0)$. Then we define the $q$-convolution of $f$ and $g$ as follows:

$$
\left(f_{1} * g\right)(t)=\int_{0}^{t} f_{1}(\tau) g(t-q \tau) d_{q} \tau,
$$

where $g(t-q \tau)=(t-q \tau)_{q}^{\beta-1}$. From (2.37), we have

$$
\begin{aligned}
\left(f_{1} * g\right)(t) & =\int_{0}^{t} \tau^{\alpha}(t-\tau q)_{q}^{\beta-1} d_{q} \tau=t \int_{0}^{1}(r t)^{\alpha}(t-q r t)_{q}^{\beta-1} d_{q} r \\
& =t^{\alpha+\beta} \int_{0}^{1} r^{\alpha}(1-q r)^{\beta-1} d_{q} r=t^{\alpha+\beta} B_{q}(\alpha+1, \beta) \\
& =\frac{\Gamma_{q}(\alpha+1) \Gamma_{q}(\beta)}{\Gamma_{q}(\alpha+\beta+1)} t^{\alpha+\beta} .
\end{aligned}
$$

Thus, by (2.38), we get

$$
\begin{aligned}
L_{q}\left(f_{1} * g\right) & =B_{q}(\alpha+1, \beta) \int_{0}^{\infty} E_{q}(-q s t) t^{\alpha+\beta} d_{q} t \\
& =B_{q}(\alpha+1, \beta) \frac{1}{s^{\alpha+\beta+1}} \Gamma_{q}(\alpha+\beta+1) \\
& =\left(\frac{\Gamma_{q}(\alpha+1)}{s^{\alpha+1}}\right)\left(\frac{\Gamma_{q}(\beta)}{s^{\beta}}\right) .
\end{aligned}
$$

By (2.8), we see that

$$
L_{q}\left(f_{1}\right)=\frac{\Gamma_{q}(\alpha+1)}{s^{\alpha+1}}, L_{q}(g)=\frac{1}{s^{\beta}} \Gamma_{q}(\beta) .
$$

Hence, by (2.39) and (2.40), we get

$$
L_{q}\left(f_{1} * g\right)=L_{q}\left(f_{1}\right) L_{q}(g)
$$

Assume that $f(t)$ is of the type such that equation (2.41). Then we have

$$
\begin{aligned}
f * g & =\int_{0}^{t}(t-\tau q)_{q}^{\beta-1} f(\tau) d_{q} \tau=t \int_{0}^{1}(t-q r t)_{q}^{\beta-1} f(r t) d_{q} r \\
& =T_{q}^{\beta}(f(t))
\end{aligned}
$$

where $g(t)=t^{\beta-1}$, and

$$
\begin{aligned}
L_{q}\left(T_{q}^{\beta} f(t)\right) & =L_{q}(f * g)=L_{q}(f) L_{q}(g) \\
& =\frac{\Gamma_{q}(\beta)}{s^{\beta}} L_{q}(f) .
\end{aligned}
$$


If $f(t)=\sum_{i} a_{i} t^{\alpha_{i}}$, then we have

$$
\begin{aligned}
L_{q}(f * g) & =\sum_{i} a_{i} L_{q}\left(t^{\alpha_{i}} * g\right) \\
& =\sum_{i} a_{i} L_{q}\left(t^{\alpha_{i}}\right) L_{q}(g) \\
& =L_{q}\left(\sum_{i} a_{i} t^{\alpha_{i}}\right) L_{q}(g) \\
& =L_{q}(f) L_{q}(g) .
\end{aligned}
$$

Therefore, by (2.44), we obtain the following theorem.

Theorem 2.6. For $f(t)=\sum_{i} a_{i} t^{\alpha_{i}}$ and $g(t)=t^{\beta-1}$, we have

$$
L_{q}(f * g)=L_{q}(f) L_{q}(g) .
$$

For $\beta=1$, let $T_{q} f(t)=T_{q}^{1} f(t)$. Then we see that

$$
\begin{aligned}
L_{q}\left(T_{q} \sin _{q} t\right) & =L_{q}\left(\sin _{q} t * 1\right)=L_{q}\left(\sin _{q} t\right) L_{q}(1) \\
& =\left(\frac{1}{s^{2}+1}\right)\left(\frac{1}{s}\right)=\frac{1}{s\left(s^{2}+1\right)}
\end{aligned}
$$

and

$$
L_{q}\left(T_{q} \sin _{q} t\right)=L_{q}\left(\int_{0}^{t} \sin _{q} \tau d_{q} \tau\right) .
$$

By (2.45) and (2.46), we get

$$
L_{q}\left(\int_{0}^{t} \sin _{q} \tau d_{q} \tau\right)=\frac{1}{s\left(s^{2}+1\right)} .
$$

Note that

$$
\begin{aligned}
& L_{q}\left(\int_{0}^{t}\left(1-\cos _{q} \tau\right) d_{q} \tau\right)=L_{q}\left(T_{q}\left(1-\cos _{q} t\right)\right) \\
= & L_{q}\left(\left(1-\cos _{q} t\right) * 1\right)=L_{q}\left(1-\cos _{q} t\right) L_{q}(1) \\
= & \left(\frac{1}{s}-\frac{s}{s^{2}+1}\right) \frac{1}{s}=\frac{1}{s^{2}\left(s^{2}+1\right)}
\end{aligned}
$$

and

$$
\begin{aligned}
& L_{q}\left(\int_{0}^{t}\left(\tau-\sin _{q} t\right) d_{q} \tau\right) \\
= & L_{q}\left(T_{q}\left(t-\sin _{q} t\right)\right)=L_{q}\left(\left(t-\sin _{q} t\right) * 1\right)=L_{q}\left(t-\sin _{q} t\right) L_{q}(1) \\
= & \left(\frac{1}{s^{2}}-\frac{1}{s^{2}+1}\right) \frac{1}{s}=\frac{1}{s^{2}\left(s^{2}+1\right)} \frac{1}{s}=\frac{1}{s^{3}\left(s^{2}+1\right)} .
\end{aligned}
$$

Therefore, by (2.48) and (2.49), we obtain the following corollary.

Corollary 2.7. For $t \geq 0$, we have

and

$$
L_{q}\left(\int_{0}^{t}\left(1-\cos _{q} \tau\right) d_{q} \tau\right)=\frac{1}{s^{2}\left(s^{2}+1\right)},
$$

$$
L_{q}\left(\int_{0}^{t}\left(\tau-\sin _{q} \tau\right) d_{q} \tau\right)=\frac{1}{s^{3}\left(s^{2}+1\right)},
$$


Let $u(t-a)$ be Heaviside function which defined by

$$
u(t-a)= \begin{cases}1 & \text { if } t \geq a \\ 0 & \text { if } 0 \leq t<a .\end{cases}
$$

Then, we have

$$
\begin{aligned}
L_{q}(u(t-a)) & =\int_{0}^{\infty} E_{q}(-q s t) u(t-a) d_{q} t=\int_{a}^{\infty} E_{q}(-q s t) d_{q} t \\
& =\int_{0}^{\infty} E_{q}(-q s t) d_{q} t-\int_{0}^{a} E_{q}(-q s t) d_{q} t \\
& =\frac{1}{s}-\int_{0}^{a} E_{q}(-q s t) d_{q} t=\frac{1}{s}-\sum_{n=0}^{\infty} \frac{(-1)^{n} q^{\left(\begin{array}{c}
n \\
2
\end{array}\right)}}{[n]_{q} !}(q s)^{n} \int_{0}^{a} t^{n} d_{q} t \\
& =\frac{1}{s}+\frac{1}{s} \sum_{n=1}^{\infty} \frac{(-1)^{n}}{[n]_{q} !} q^{\left(\begin{array}{c}
n \\
2
\end{array}\right)} s^{n} a^{n}=\frac{1}{s} \sum_{n=0}^{\infty} \frac{(-1)^{n} q^{\left(\begin{array}{c}
n \\
2
\end{array}\right)}}{[n]_{q} !}(a s)^{n} \\
& =\frac{1}{s} E_{q}(-q a s) .
\end{aligned}
$$

Therefore, by (2.51), we obtain the following theorem.

Theorem 2.8. Let $u(t-a)$ be Heaviside function. Then we have

$$
L_{q}(u(t-a))=\frac{1}{s} E_{q}(-q a s) .
$$

\section{3. $q$-LAPLACE TRANSFORM OF THE SECOND KIND}

In this section, we consider the $q$-Laplace transform with $e_{q}(-s t)$ and is called $q$-Laplace transform of the second kind. The $q$-Laplace transform of the second kind is defined by

$$
\tilde{F}(s)=\tilde{L}_{q}(f(t))=\int_{0}^{\infty} e_{q}(-s t) f(t) d_{q} t,(s>0) .
$$

As is well known, the q-gamma function of the second kind is defined by

$$
\gamma_{q}(t)=\int_{0}^{\infty} x^{t-1} e_{q}(-x) d_{q} x,(t>0), \quad(\text { see }[2,6]) .
$$

Thus, by (3.2), we get

$$
\gamma_{q}(1)=1, \gamma_{q}(t+1)=q^{-t}[t]_{q} \gamma_{q}(t), \gamma_{q}(n)=q^{-\left(\begin{array}{c}
n \\
2
\end{array}\right)} \Gamma_{q}(n),(n \in \mathbb{N}) .
$$

For $\alpha \in \mathbb{R}$ with $\alpha>-1$, we have

$$
\begin{aligned}
\tilde{L}_{q}\left(t^{\alpha}\right) & =\int_{0}^{\infty} e_{q}(-s t) t^{\alpha} d_{q} t=\frac{1}{s^{\alpha+1}} \int_{0}^{\infty} e_{q}(-t) t^{\alpha} d_{q} t \\
& =\frac{1}{s^{\alpha+1}} \gamma_{q}(\alpha+1) .
\end{aligned}
$$

In particular, for $\alpha=n \in \mathbb{N}$, by (3.3) and (3.4), we get

$$
\begin{aligned}
\tilde{L}_{q}\left(t^{n}\right) & =\frac{1}{s^{n+1}} \gamma_{q}(n+1)=\frac{1}{s^{n+1}} q^{-\left(\begin{array}{c}
n+1 \\
2
\end{array}\right)} \Gamma_{q}(n+1) \\
& =\frac{1}{s^{n+1}} q^{-\frac{(n+1) n}{2}}[n]_{q} ! .
\end{aligned}
$$


Let us take $\alpha=-\frac{1}{2}$. Then we have

$$
\tilde{L}_{q}\left(t^{-\frac{1}{2}}\right)=\frac{1}{\sqrt{s}} \gamma_{q}\left(\frac{1}{2}\right), \tilde{L}_{q}\left(t^{\frac{1}{2}}\right)=\frac{1}{\sqrt{s^{3}} q[2]_{q^{\frac{1}{2}}}} \gamma_{q}\left(\frac{1}{2}\right) .
$$

In a similar way, we can give the $q$-Laplace transform of the second kind for $e_{q}(a t)$ and $E_{q}(a t)$.

$$
\begin{aligned}
\tilde{L}_{q}\left(e_{q}(a t)\right) & =\sum_{n=0}^{\infty} \frac{a^{n}}{[n]_{q} !} \int_{0}^{\infty} e_{q}(-s t) t^{n} d_{q} t=\sum_{n=0}^{\infty} \frac{a^{n}}{[n]_{q} !} \tilde{L}_{q}\left(t^{n}\right) \\
& =\sum_{n=0}^{\infty} \frac{a^{n}}{[n]_{q} !} \frac{q^{-\left(\begin{array}{c}
n+1 \\
2
\end{array}\right)}}{s^{n+1}} \Gamma_{q}(n+1)=\sum_{n=0}^{\infty} \frac{a^{n}}{s^{n+1}} q^{-\left(\begin{array}{c}
n+1 \\
2
\end{array}\right)},
\end{aligned}
$$

and

$$
\begin{aligned}
\tilde{L}_{q}\left(E_{q}(a t)\right) & =\sum_{n=0}^{\infty} \frac{q^{\left(\begin{array}{c}
n \\
2
\end{array}\right)} a^{n}}{[n]_{q} !} \int_{0}^{\infty} t^{n} e_{q}(-s t) d_{q} t=\sum_{n=0}^{\infty} \frac{q^{\left(\begin{array}{c}
n \\
2
\end{array}\right)} a^{n}}{[n]_{q} !} \tilde{L}_{q}\left(t^{n}\right) \\
& =\sum_{n=0}^{\infty} \frac{q^{\left(\begin{array}{c}
n \\
2
\end{array}\right)} a^{n}}{[n]_{q} !} \frac{q^{-\left(\begin{array}{c}
n+1 \\
2
\end{array}\right)}}{s^{n+1}} \Gamma_{q}(n+1)=\frac{1}{s} \sum_{n=0}^{\infty}\left(\frac{a}{q s}\right)^{n} \\
& =\frac{1}{s}\left(\frac{q s}{q s-a}\right)=\frac{q}{q s-a} .
\end{aligned}
$$

Therefore, by (3.4)-(3.8), we obtain the following theorem.

Theorem 3.1. For $\alpha \in \mathbb{R}$ with $\alpha>-1$, we have

$$
\tilde{L}_{q}\left(t^{\alpha}\right)=\frac{1}{s^{\alpha+1}} \gamma_{q}(\alpha+1),(s>0) .
$$

In particular, if $\alpha=n \in \mathbb{N}$, then we see that

$$
\tilde{L}_{q}\left(t^{n}\right)=\frac{1}{s^{n+1}} q^{-\left(\begin{array}{c}
n+1 \\
2
\end{array}\right)}[n]_{q} !
$$

Moreover,

$$
\tilde{L}_{q}\left(e_{q}(a t)\right)=\sum_{n=0}^{\infty} \frac{a^{n}}{s^{n+1}} q^{-\left(\begin{array}{c}
n+1 \\
2
\end{array}\right)}, \tilde{L}_{q}\left(E_{q}(a t)\right)=\frac{q}{q s-a},
$$

and

$$
\tilde{L}_{q}\left(t^{-\frac{1}{2}}\right)=\frac{1}{\sqrt{s}} \gamma_{q}\left(\frac{1}{2}\right), \tilde{L}_{q}\left(t^{\frac{1}{2}}\right)=\frac{1}{\sqrt{s^{3}} q[2]_{q^{\frac{1}{2}}}} \gamma_{q}\left(\frac{1}{2}\right) .
$$

From (1.1), we note that

$$
E_{q}(i t)=\sum_{n=0}^{\infty} \frac{q^{\left(\begin{array}{c}
n \\
2
\end{array}\right)}}{[n]_{q} !} i^{n} t^{n}
$$

Thus, by (3.9), we get

$$
E_{q}(i t)=\sum_{n=0}^{\infty} \frac{(-1)^{n} q^{\left(\begin{array}{c}
2 n \\
2
\end{array}\right)}}{[2 n]_{q} !} t^{2 n}+i \sum_{n=0}^{\infty} \frac{(-1)^{n} q^{\left(\begin{array}{c}
2 n+1 \\
2
\end{array}\right)}}{[2 n+1]_{q} !} t^{2 n+1} .
$$


By (3.10), we can define new $q$-Sine and $q$-Cosine function as follows:

$$
\operatorname{Cos}_{q}(a t)=\sum_{n=0}^{\infty} \frac{q^{\left(\begin{array}{c}
2 n \\
2
\end{array}\right)}(-1)^{n} a^{2 n}}{[2 n]_{q} !} t^{2 n}=\frac{1}{2}\left[E_{q}(i a t)+E_{q}(-i a t)\right],
$$

and

$$
\operatorname{Sin}_{q}(a t)=\sum_{n=0}^{\infty} \frac{q^{\left(\begin{array}{c}
2 n+1 \\
2
\end{array}\right)}(-1)^{n} a^{2 n+1}}{[2 n+1]_{q} !} t^{2 n+1}=\frac{1}{2}\left[E_{q}(i a t)-E_{q}(-i a t)\right] .
$$

Now, we give the $q$-Laplace transform of the second kind for $q$-Sine and $q$-Cosine functions:

From (2.16) and (3.7), we note that

$$
\begin{aligned}
\tilde{L}_{q}\left(\cos _{q} a t\right) & =\frac{1}{2}\left[\tilde{L}_{q}\left(e_{q}(i a t)\right)+\tilde{L}_{q}(-i a t)\right] \\
& =\frac{1}{2}\left[\sum_{I=0}^{\infty} \frac{q^{-\left(\begin{array}{c}
n+1 \\
2
\end{array}\right)}}{s^{n+1}}(i a)^{n}+\sum_{n=0}^{\infty} \frac{q^{-\left(\begin{array}{c}
n+1 \\
2
\end{array}\right)}}{s^{n+1}}(-i a)^{n}\right] \\
& =\frac{1}{2} \times 2 \sum_{n=0}^{\infty} \frac{q^{-n(2 n+1)}}{s^{2 n+1}}\left(\frac{a}{s}\right)^{2 n}=\frac{1}{s} \sum_{n=0}^{\infty}(-1)^{n} q^{-n(2 n+1)}\left(\frac{a}{s}\right)^{2 n},
\end{aligned}
$$

and

$$
\begin{aligned}
\tilde{L}_{q}\left(\sin _{q} a t\right) & =\sum_{n=0}^{\infty} \frac{(-1)^{n} a^{2 n+1}}{[2 n+1]_{q} !} \int_{0}^{\infty} e_{q}(-s t) t^{2 n+1} d_{q} t=\sum_{n=0}^{\infty} \frac{(-1)^{n} a^{2 n+1}}{[2 n+1]_{q} !} \tilde{L}_{q}\left(t^{2 n+1}\right) \\
& =\sum_{n=0}^{\infty} \frac{(-1)^{n} a^{2 n+1}}{[2 n+1]_{q} !} \times \frac{[2 n+1]_{q} !}{s^{2 n+1}} q^{-\frac{(2 n+1)(2 n+2)}{2}} \\
& =\frac{1}{s} \sum_{n=0}^{\infty}(-1)^{n} q^{-(n+1)(2 n+1)}\left(\frac{a}{s}\right)^{2 n+1}
\end{aligned}
$$

Therefore, by (3.13) and (3.14), we obtain the following theorem.

Theorem 3.2 (Transforms of $q$-cosine and $q$-sine).

$$
\begin{gathered}
\tilde{L}_{q}\left(\cos _{q} a t\right)=\frac{1}{s} \sum_{n=0}^{\infty}(-1)^{n} q^{-n(2 n+1)}\left(\frac{a}{s}\right)^{2 n}, \\
\tilde{L}_{q}\left(\sin _{q} a t\right)=\frac{1}{s} \sum_{n=0}^{\infty}(-1)^{n} q^{-(n+1)(2 n+1)}\left(\frac{a}{s}\right)^{2 n+1} .
\end{gathered}
$$

We consider $q$-Laplace transforms of the second kind for the $q$-Cosine and $q$-Sine functions of the second kind. By (3.11) and (3.12), we get

$$
\begin{aligned}
\tilde{L}_{q}\left(\operatorname{Cos}_{q}(a t)\right) & =\sum_{n=0}^{\infty} \frac{q^{\left(\begin{array}{c}
2 n \\
2
\end{array}\right)}(-1)^{n} a^{2 n}}{[2 n]_{q} !} \int_{0}^{\infty} t^{2 n} e_{q}(-s t) d_{q} t \\
& =\sum_{n=0}^{\infty} \frac{q^{\left(\begin{array}{c}
2 n \\
2
\end{array}\right)}(-1)^{n} a^{2 n}}{[2 n]_{q} !} \times \frac{[2 n]_{q} !}{s^{2 n+1}} q^{-\left(\begin{array}{c}
2 n+1 \\
2
\end{array}\right)} \\
& =\frac{1}{s} \sum_{n=0}^{\infty}\left(\frac{a}{q s}\right)^{2 n}(-1)^{n}=\frac{q^{2} s}{(q s)^{2}+a^{2}}
\end{aligned}
$$


and

$$
\begin{aligned}
\tilde{L}_{q}\left(\operatorname{Sin}_{q}(a t)\right) & =\frac{1}{2 i}\left\{\tilde{L}_{q}\left(E_{q}(i a t)\right)-\tilde{L}_{q}\left(E_{q}(-i a t)\right)\right\} \\
& =\frac{1}{2 i}\left\{\frac{q}{q s-i a}-\frac{q}{q s+i a}\right\}=\frac{1}{2 i} \times \frac{2 q i a}{(q s)^{2}+a^{2}}=\frac{q a}{(q s)^{2}+a^{2}} .
\end{aligned}
$$

Therefore, by (3.16) and (3.17), we obtain the following theorem.

Theorem 3.3 (Transform of $q$-Sine and $q$-Cosine function).

$$
\tilde{L}_{q}\left(\operatorname{Cos}_{q}(a t)\right)=\frac{q^{2} s}{(q s)^{2}+a^{2}}, \tilde{L}_{q}\left(\operatorname{Sin}_{q}(a t)\right)=\frac{q a}{(q s)^{2}+a^{2}} .
$$

Here, we compute the $q$-Laplace transform of derivative by using the definition of the $q$-Laplace transform of the second kind. It is easy to show that

$$
\int_{0}^{x} f^{\prime}(t) g(t) d_{q} t=(f(x) g(t)-f(0) g(0))-\int_{0}^{x} f(q t) g^{\prime}(t) d_{q} t,
$$

where $f^{\prime}(t)=D_{q} f(t)=\frac{\partial_{q} f(t)}{\partial_{q} t}$.

By (1.2), (3.1) and (3.18), we get

$$
\tilde{L}_{q}\left(f^{\prime}(t)\right)=\int_{0}^{\infty} f^{\prime}(t) e_{q}(-s t) d_{q} t=-f(0)-\int_{0}^{\infty} f(q t) e_{q}^{\prime}(-s t) d_{q} t .
$$

From (1.2), we note that

$$
\begin{aligned}
e_{q}^{\prime}(-s t) & =\frac{\partial_{q}}{\partial_{q} t} e_{q}(-s t)=\sum_{n=0}^{\infty} \frac{(-1)^{n}}{[n]_{q} !} s^{n} \frac{\partial_{q} t^{n}}{\partial_{q} t}=\sum_{n=1}^{\infty} \frac{(-1)^{n} s^{n}}{[n-1]_{q} !} t^{n-1} \\
& =-s \sum_{n=0}^{\infty} \frac{(-1)^{n}}{[n]_{q} !} s^{n} t^{n}=-s e_{q}(-s t) .
\end{aligned}
$$

By (3.19) and (3.20), we get

$$
\begin{aligned}
\tilde{L}_{q}\left(f^{\prime}(t)\right) & =-f(0)+s \int_{0}^{\infty} f(q t) e_{q}(-s t) d_{q} t \\
& =-f(0)+s q^{-1} \int_{0}^{\infty} f(t) e_{q}\left(-q^{-1} s t\right) d_{q} t \\
& =-f(0)+s q^{-1} \tilde{F}\left(q^{-1} s\right),
\end{aligned}
$$

where $f^{\prime}(t)=D_{q} f(t)=\frac{\partial_{q} f(t)}{\partial_{q} t}$. If we replace $f(t)$ by $D_{q} f(t)=\frac{\partial_{q} f(t)}{\partial_{q} t}$, then we have

$$
\tilde{L}_{q}\left(f^{(2)}(t)\right)=s^{2} q^{-3} \tilde{F}\left(q^{-2} s\right)-q^{-1} s f(0)-f^{\prime}(0)
$$

and

$$
\tilde{L}_{q}\left(f^{(3)}(t)\right)=s^{3} q^{-6} \tilde{F}\left(q^{-3} s\right)-s^{2} q^{-3} f(0)-s q^{-1} f^{\prime}(0)-f^{(2)}(0),
$$

where $f^{(3)}(t)=D_{q}^{3} f(t)=\left(\frac{\partial_{q}}{\partial_{q} t}\right)^{3} f(t), f^{(2)}(0)=\left.\left(\frac{\partial_{q}}{\partial_{q} t}\right)^{2} f(t)\right|_{t=0}$. Continuing this process, we get

$$
\tilde{L}_{q}\left(f^{(n)}(t)\right)=s^{n} q^{-\left(\begin{array}{c}
n+1 \\
2
\end{array}\right)} \tilde{F}\left(q^{-n} s\right)-\sum_{i=0}^{n-1} s^{n-1-i} q^{-\left(\begin{array}{c}
n-i \\
2
\end{array}\right)} f^{(i)}(0),
$$


where $f^{(n)}(t)=D_{q}^{n} f(t)=\left(\frac{\partial_{q}}{\partial_{q} t}\right)^{n} f(t), f^{(n)}(0)=\left.\left(\frac{\partial_{q}}{\partial_{q} t}\right)^{n} f(t)\right|_{t=0}$. Therefore, by (3.23), we obtain the following theorem.

Theorem 3.4. For $n \in \mathbb{N}$, we have

$$
\tilde{L}_{q}\left(f^{(n)}(t)\right)=s^{n} q^{-\left(\begin{array}{c}
n+1 \\
2
\end{array}\right)} \tilde{F}\left(q^{-n} s\right)-\sum_{i=0}^{n-1} s^{n-1-i} q^{-\left(\begin{array}{c}
n-i \\
2
\end{array}\right)} f^{(i)}(0)
$$

where $\tilde{F}(s)=\tilde{L}_{q}(f(t))=\int_{0}^{\infty} e_{q}(-s t) f(t) d_{q} t, f^{(n)}(t)=\left(\frac{\partial_{q}}{\partial_{q} t}\right)^{n} f(t)$.

Let us consider the following $s$-derivative for the $q$-Laplace transform of the second kind.

$$
\begin{aligned}
\frac{\partial_{q} \tilde{F}(s)}{\partial_{q} s} & =\frac{\partial_{q} \tilde{L}_{q}(f(t))}{\partial_{q} s}=\frac{\partial_{q}}{\partial_{q} s} \int_{0}^{\infty} e_{q}(-s t) f(t) d_{q} t \\
& =\int_{0}^{\infty}\left(-t e_{q}(-s t)\right) f(t) d_{q} t=-\tilde{L}_{q}(t f(t))
\end{aligned}
$$

Thus, by (3.24), we get

$$
\tilde{L}_{q}(t f(t))=-\frac{\partial_{q}}{\partial_{q} s} \tilde{F}(s)
$$

Continuing this process, we get

$$
\tilde{L}_{q}\left(t^{n} f(t)\right)=(-1)^{n}\left(\frac{\partial_{q}}{\partial_{q} s}\right)^{n} \tilde{F}(s) .
$$

Therefore, by (3.26), we obtain the following theorem.

Theorem 3.5. For $n \in \mathbb{N}$, we have

$$
\tilde{L}_{q}\left(t^{n} f(t)\right)=(-1)^{n}\left(\frac{\partial_{q}}{\partial_{q} s}\right)^{n} \tilde{F}(s) .
$$

For example, if we take $f(t)=e_{q}(a t)$, then

$$
\begin{aligned}
\tilde{L}_{q}\left(t^{n} e_{q}(a t)\right) & =(-1)^{n}\left(\frac{\partial_{q}}{\partial_{q} s}\right)^{n} \tilde{L}_{q}\left(e_{q}(a t)\right)=(-1)^{n}\left(\frac{\partial_{q}}{\partial_{q} s}\right)^{n} \sum_{k=0}^{\infty} \frac{q^{-\frac{k(k+1)}{2}}}{s^{k+1}} a^{k} \\
& =(-1)^{n} \sum_{k=0}^{\infty} a^{k}(-1)^{k} q^{-n k-\left(\begin{array}{c}
k+1 \\
2
\end{array}\right)-\left(\begin{array}{c}
n+1 \\
2
\end{array}\right)} \times \frac{[k+1]_{q}[k+2]_{q} \cdots[k+n]_{q}}{s^{k+n+1}},
\end{aligned}
$$

and

$$
\begin{aligned}
\tilde{L}_{q}\left(t^{n} E_{q}(a t)\right) & =(-1)^{n}\left(\frac{\partial_{q}}{\partial_{q} s}\right)^{n} \tilde{L}_{q}\left(E_{q}(a t)\right)=(-1)^{n}\left(\frac{\partial_{q}}{\partial_{q} s}\right)^{n} \frac{q}{q s-a} \\
& =(-1)^{n}(-1)^{n} q^{n+1}[n]_{q} ! \frac{1}{(q s-a)\left(q^{2} s-a\right) \cdots\left(q^{n+1} s-a\right)} \\
& =q^{n+1} \frac{[n]_{q} !}{(q s-a)\left(q^{2} s-a\right) \cdots\left(q^{n+1} s-a\right)} .
\end{aligned}
$$


Finally, let us consider

$$
\begin{aligned}
L_{q}\left(e_{q}(a t) f(t)\right) & =\int_{0}^{\infty} e_{q}(-s t) e_{q}(a t) f(t) d_{q} t \\
& =\sum_{n=0}^{\infty} \frac{a^{n}}{[n]_{q} !} \int_{0}^{\infty} e_{q}(-s t) t^{n} f(t) d_{q} t \\
& =\sum_{n=0}^{\infty} \frac{a^{n}}{[n]_{q} !}(-1)^{n} \tilde{L}_{q}\left(t^{n} f(t)\right) \\
& =\sum_{n=0}^{\infty} \frac{a^{n}}{[n]_{q} !}(-1)^{n}\left(\frac{\partial_{q}}{\partial_{q} s}\right)^{n} \tilde{L}_{q}(f(t)) \\
& =\sum_{n=0}^{\infty} \frac{a^{n}}{[n]_{q} !}(-1)^{n}\left(\frac{\partial_{q}}{\partial_{q} s}\right)^{n} \tilde{F}(s) .
\end{aligned}
$$

\section{REFERENCES}

[1] G. Andrews, R. Askey and R. Roy, Special functions, Cambridge University Press, Cambridge, 1999.

[2] R. Diaz and C. Teruel, q,k-generalized gamma and beta functions, J. Nonlinear Math. Phys. 12 (2005), no. 1, 118-134.

[3] T. Mansour and A. S. Shabani, Generalization of some inequalities for the $\left(q_{1}, \cdots, q_{s}\right)$ gamma function, Matematiche (Catania), 67 (2012), o. 2, 119-130.

[4] M. Mansour, Determining the $k$-generalized gamma function $\Gamma_{k}(x)$ by functional equations, Int. J. Contemp. Math. Sci., 4 (2009), no. 21-24, 1037-1042.

[5] T. Mansour, Some inequalities for the q-gamma function, J. Inequal. Pure Appl. Math., 9 (2008) no. 1, Article 18, 4 pp.

[6] A. de Sole and V. Kac, On integral representations of q-gamma and q-beta functions, arXiv: math. QA/0302032, 2003.

[7] F. Ucar and D. Albayrak, On q-Laplace type integral operators and their applications, J. Difference Equ. Appl., 18 (2012), no. 6, 1001-1014.

[8] R. K. Yadav, S. O. Purohit and P. Nirwan, On q-Laplace transforms of a general class of q-polynomials and q-hypergeometric functions, Math. Maced., 7 (2009), 81-88.

[9] D. G. Zill and M. R. Cullen, Advanced Engineering Mathematics, ones and Bartlett, 2005.

1 Department of Physics, Gyeongsang National University, Jinju 660,701, Republic OF KOREA.

E-mail address: mimip4444@hanmail.net

2 Department of Mathematics, Kwangwoon University, Seoul 139-701, Republic of KOREA.

E-mail address: tkkim@kw.ac.kr 